\title{
AVALIAÇÃo dOS TEORES DE Pb, Cd, Sn, Co, Hg, Mo E As EM SOLOS DA PENÍNSUla FILDES - ANTÁRTICA
}

\author{
Bruna M. Dalfior ${ }^{a}$, Larissa D. Roriz ${ }^{\text {a }}$, Reginaldo F. Júnior ${ }^{\mathrm{a}}$, Antônio Carlos de Freitas ${ }^{\mathrm{c}}$, Heitor E. da Silva ${ }^{\mathrm{c}}$, Maria Tereza \\ W.D. Carneiro ${ }^{a}$, Marcus Vinícius V. J. Licinio ${ }^{\mathrm{b}}$ e Geisamanda P. Brandão ${ }^{\mathrm{a}}$,* $^{-}$ \\ aDepartamento de Química, Centro de Ciências Exatas, Universidade Federal do Espírito Santo, 29075-910 Vitória - ES, Brasil \\ ${ }^{\text {b}}$ Departamento de Ciências Fisiológicas, Centro de Ciências da Saúde, Universidade Federal do Espírito Santo, 29043-900 Vitória \\ - ES, Brasil \\ ${ }^{\mathrm{c}}$ Departamento de Biofísica e Biometria, Instituto de Biologia, Universidade do Estado do Rio de Janeiro, 20550-900 Rio de \\ Janeiro - RJ, Brasil
}

Recebido em 08/10/2015; aceito em 10/06/2016; publicado na web em 01/08/2016

\begin{abstract}
EVALUATION OF Pb, Cd, Sn, Co, Hg, Mo AND As IN SOIL FROM FILDES PENINSULA - ANTARCTICA. Antarctic Continent is one of the few places that has not been explored at all, but studies show that this place is suffering interference by human activities. Therefore, monitoring of this region becomes very important. After optimization and verification of the methodology, the concentrations of $\mathrm{Pb}, \mathrm{Cd}, \mathrm{Sn}, \mathrm{Co}, \mathrm{Hg}$, Mo and As in soil samples collected in the Fildes Peninsula, King George Island, Antarctica, were determined by ICP-MS. The ranges found $\left(\mu \mathrm{g} \mathrm{kg}^{-1}\right)$ were: for $\mathrm{Pb} 525.2-2314.1$, for Cd 54.8-193.8, for Sn 54.8-193.8, for Co 2095.0-11094.1, for $\mathrm{Hg}<2.4-54.6$, for Mo 5.3-38.7 and for As 120.3-1297.1. In general, the presence of these elements can be attributed to different factors, such as natural composition of soil, melting process and transport of chemical elements caused by physical phenomena as sea and air currents, besides anthropic contamination. Using enrichment factor was possible suggest contribution by anthropic activities mainly for Co and As. Thus, it is important monitoring the elements levels constantly assisting in environmental protection.
\end{abstract}

Keywords: soil; Antarctica; trace elements; ICP-MS.

\section{INTRODUÇÃO}

A Antártica, devido ao seu isolamento geográfico e condições climáticas extremas é um dos ambientes mais preservados do mundo e por muito tempo foi considerado um ambiente controle, porém, estudos mostram que essa região não tem escapado do impacto das atividades antrópicas. ${ }^{1-3}$

Devido ao isolamento, os bens naturais desse continente demoraram um tempo para ser explorados. Foi a partir de 1900 que as Ilhas Antárticas começaram a ser exploradas principalmente para a pesca de baleias e outros animais marinhos, o que provocou o transporte e introdução de espécies animais e vegetais exóticas, acarretando um desequilíbrio ambiental. Além disso, algumas espécies de animais marinhos da região estiveram próximos à extinção. ${ }^{1,4}$

Nos últimos 50 anos a Antártica tem se tornado um espaço de estudos científicos, com a implantação de estações de pesquisa de diversas nacionalidades. Atualmente existem cerca de 79 estações científicas nas Ilhas Antárticas, sendo uma parte permanente e outra somente em funcionamento no período do verão. ${ }^{5}$

$\mathrm{O}$ crescimento das atividades antrópicas nesse continente impulsionou a regulamentação de normas que visam à diminuição dos impactos causados pela ocupação humana. Com base nessas normas foi assinado em 1991 o Protocolo de Proteção Ambiental do Tratado da Antártica, principal documento internacional que regulamenta as atividades na Antártica, ficando estabelecido que este território se caracteriza como uma reserva natural destinada à paz e à ciência, excluindo todo e qualquer tipo de exploração. ${ }^{6}$

Além da contaminação causada pelas estações científicas, pesquisadores tem se empenhado em estudar a poluição causada a partir da absorção de poluentes provenientes do transporte de partículas pelas correntes de ar e marítimas em grande escala

*e-mail: geisamanda@gmail.com geográfica, originados das atividades humanas desenvolvidas em outros continentes. ${ }^{6,7}$

Dentro do continente Antártico existem locais mais vulneráveis à contaminação por transporte devido a sua proximidade com a América do Sul, como é o caso do Norte da Península Antártica e algumas ilhas ao redor, dentre elas a Ilha Rei George. Existem registros de atividade humana nessa Ilha desde o século XIX e atualmente na Ilha Rei George funcionam nove estações de pesquisa permanente e uma pista de pouso, o que evidencia alta presença humana, sendo que suas atividades podem causar efeitos diversos nesse ambiente. ${ }^{1,8}$

Diversas pesquisas vêm sendo desenvolvidas com amostras da Ilha Rei George com a finalidade de monitorar os efeitos das atividades humanas local e global nesse ambiente e nos seres que nele habitam.

Apesar dos elementos traço serem de ocorrência natural no meio ambiente, o crescimento da atividade humana tem proporcionado um aumento nos níveis desses elementos na natureza, podendo acarretar danos à saúde humana, à fauna e à flora. Portanto, a determinação dos níveis de elementos traço em diversas matrizes tem se tornado uma tarefa importante. ${ }^{9-11}$

O monitoramento de elementos traço em algumas espécies de pinguins da Ilha Rei George e da Ilha Decepção sugeriu a existência de uma relação entre o aumento dos níveis de elementos como $\mathrm{Cr}, \mathrm{Ni}$, $\mathrm{Pb}, \mathrm{Mn}, \mathrm{Cd}$ e As e as atividades antrópicas, sendo estas as possíveis fontes de contaminação. ${ }^{8}$

Estudos como o de Lu e colaboradores ${ }^{12}$ relatam os teores de alguns elementos em amostras de solo da Península Fildes, Ilha Rei George, Antártica. As amostras foram coletadas em uma expedição de verão em 2009/2010 em pontos próximos a três estações de pesquisa. As análises permitiram concluir que as concentrações de $\mathrm{Pb}, \mathrm{Cd}$ e $\mathrm{Hg}$ foram consideravelmente aumentadas pela atividade humana. 
Licínio e colaboradores ${ }^{13}$ propuseram uma reconstrução temporal da contaminação de elementos traço na Baía Almirantado, Antártica. Foi detectado um aumento no perfil dos sedimentos em relação aos elementos $\mathrm{Cr}$, $\mathrm{Cu}$ e Ni, que pode estar relacionado ao uso de petróleo e tintas, uma vez que as amostras analisadas foram coletadas nas proximidades da estação de pesquisa.

Dentre as matrizes que vem sendo utilizadas em estudos de monitoramento ambiental destacam-se: solo, água, plantas bioindicadoras e organismos de ambientes marinhos.

O solo tem sido muito estudado uma vez que ele pode indicar a condição do ambiente, por possuir a capacidade de agir como um reservatório para esses elementos. Além disso, ele apresenta estabilidade físico-química e características decorrentes da geologia da área em que se encontra. ${ }^{12-14}$

A determinação dos elementos traço nestas matrizes ambientais contribui na avaliação da disponibilidade desses contaminantes para os seres vivos que habitam o local e no controle dos impactos das atividades antrópicas. ${ }^{2,3,15}$ Diante disso faz-se necessário o uso de técnicas analíticas que sejam adequadas para a avaliação e monitoramento dos teores dos elementos traço.

Os métodos analíticos mais difundidos para determinação de elementos traço em matrizes ambientais envolvem a espectrometria de emissão óptica com plasma indutivamente acoplado (ICP OES), espectrometria de massa com plasma indutivamente acoplado (ICP-MS), espectrometria de absorção atômica em chama (F AAS), espectrometria de absorção atômica por geração de hidreto (HG AAS), espectrometria de absorção atômica em forno de grafite (GF AAS), entre outras técnicas. ${ }^{16,17} \mathrm{~A}$ absorção atômica é uma técnica muito difundida e apresenta poucas interferências de matriz, porém, geralmente é monoelementar. Já o ICP OES se caracteriza por ser uma técnica multielementar, porém, quando comparado ao ICP-MS, tem menor sensibilidade e seletividade. ${ }^{15,18}$ Diante disso a espectrometria de massa com plasma indutivamente acoplado tem se tornado cada vez mais atraente para análises em matrizes ambientais por ser uma análise rápida, multielementar, de alta sensibilidade e seletividade. ${ }^{18}$

Assim, o presente trabalho objetivou quantificar os teores de $\mathrm{Pb}$, $\mathrm{Cd}, \mathrm{Sn}, \mathrm{Co}, \mathrm{Hg}$, Mo e As em amostras de solo da Ilha Rei George, Península Fildes, Antártica, correlacionar esses teores entre si e comparar com valores já reportados na literatura. A partir desses resultados foram propostas as possíveis fontes e atividades humanas que podem contribuir para a presença desses elementos. Para tanto, a quantificação foi realizada por espectrometria de massa com plasma indutivamente acoplado (ICP-MS).

\section{PARTE EXPERIMENTAL}

\section{Reagentes e equipamentos}

Para o preparo das soluções e também descontaminação de todos os materiais foi utilizada água purificada com resistividade de 18,2 $\mathrm{M} \Omega$, purificada em sistema PURELAB Ultra (ELGA, UK).

Todos os materiais utilizados no estudo passaram por lavagem com água e banho próprio para descontaminação por 48 horas utilizando Extran MA 02 Neutro (Merck, Darmstadt, Alemanha), procedimento padrão do Laboratório de Espectrometria Atômica (LEA) do LabPetro/Ufes. Após esse período o material foi lavado com água deionizada e colocado em banho ácido $\left(\mathrm{HNO}_{3} 20 \% \mathrm{v} / \mathrm{v}\right)$ por no mínimo 24 horas. Posteriormente os materiais foram lavados com água purificada, secos e armazenados em local isento de metais para evitar que houvesse contaminação, que foi avaliada pelo controle do branco.

Para os procedimentos de preparo de amostra foram utilizados $\mathrm{HNO}_{3} 68 \%$ m/m P.A (Neon Comercial Ltda.) e $\mathrm{HCl} 37 \%$ m/m
P.A (Veritas® GFS Chemicals, Inc.), purificados por destilação no Distillacid BSB939 IV (Berghof, Alemanha). Utilizaram-se soluções monoelementar $1000 \mathrm{mg} \mathrm{L}^{-1}$ de Pb, Cd, Sn, Co, Hg, Mo e As (SCP Science, Canadá) onde foram realizadas diluições necessárias para o preparo de uma solução contendo todos esses elementos. Foram usados no teste de padronização interna os elementos ródio ( $\mathrm{Rh}, 5 \mu \mathrm{g}$ $\mathrm{L}^{-1}$ ), lutércio ( $\mathrm{Lu}, 5 \mu \mathrm{g} \mathrm{L}^{-1}$ ) e índio ( $\mathrm{In}, 5 \mu \mathrm{g} \mathrm{L}{ }^{-1}$ ) a partir de padrões monoelementares de concentração $1000 \mathrm{mg} \mathrm{L}^{-1}$ (SPC Science, Canadá).

Os equipamentos utilizados no preparo da amostra e na análise foram: agitador mecânico (Modelo 500-2D, Nova Ética, Brasil); balança analítica ED224S (Sartorius tecnologia de pesagem, Goettingen, Alemanha) com precisão de $0,0001 \mathrm{~g}$; e espectrômetro de massas com plasma indutivamente acoplado ICP-MS (NexIon 300D, Perkin Elmer, Estados Unidos). Foi utilizado para a geração/manutenção do plasma do ICP-MS, aspiração da amostra e gás auxiliar o gás argônio premier com pureza de 99,9992\% (Air Products Brasil Ltda, São Paulo).

Para realizar os cálculos de fator de enriquecimento foi utilizado como elemento normalizador o $\mathrm{Fe}$, que foi determinado em espectrômetro de absorção atômica por chama F AAS (ZEEnit 700, Analytic Jena, Alemanha). Utilizou-se uma lâmpada de catodo oco de Fe (Analytic Jena, Alemanha), linha de absorção em 248,3 nm e chama composta por acetileno (99,5\%, Air Products Brasil Ltda, São Paulo) e ar sintético (99,9\%, Air Products Brasil Ltda, São Paulo).

\section{Coleta das amostras}

Durante a expedição do Programa ProAntar que ocorreu no período de 03 a 29 de março de 2013 foram realizadas coletas de solo ao longo de toda a extensão da Península Fildes, Ilha Rei George, Antártica. Para assegurar a homogeneidade da amostra, para cada ponto de coleta foi realizada uma amostragem composta na qual 5 alíquotas foram coletadas na superfície em um círculo de um metro de diâmetro. As coletas foram realizadas com espátulas de teflon, previamente lavadas com $\mathrm{HCl} 10 \%$ v/v e o solo coletado foi acondicionado em sacos plásticos e armazenados em geladeira para posterior análise no Brasil. A Figura 1 apresenta os pontos onde foram realizadas as coletas.

\section{Caracterização do solo}

Levando em consideração a importância da granulometria e do $\mathrm{pH}$ dos solos na disponibilidade dos elementos traço para o ambiente, foi analisado a fração do solo com granulometria inferior a 10 mesh e avaliou-se o $\mathrm{pH}$ seguindo a metodologia descrita por Amaral e colaboradores. ${ }^{19}$

\section{Preparo das amostras de solo para a determinação elementar}

As amostras de solo passaram por secagem em estufa a $60^{\circ} \mathrm{C}$ até peso constante e posteriormente foram homogeneizadas e peneiradas em três telas de nylon de diferentes diâmetros, sendo que somente a fração menor que 10 mesh foi analisada, conforme descrito na norma ASTM D3974-09. ${ }^{20}$ As amostras coletadas nos pontos P5, P7 e P12 após homogeneização não apresentaram material com granulometria inferior a 10 mesh, portanto não foram analisadas. As amostras peneiradas foram armazenadas em sacolas descontaminadas e hermeticamente fechadas.

O pré tratamento das amostras de solo utilizado para realizar a extração dos elementos foi conduzido de acordo com a norma ASTM D3974-0920 prática B modificada: pesou-se 0,5 g de amostra em um erlenmeyer de $125 \mathrm{~mL}$; adicionou-se $47,5 \mathrm{~mL}$ de $\mathrm{H}_{2} \mathrm{O}$ e $2,5 \mathrm{~mL}$ de $\mathrm{HCl}$ purificado; a mistura foi colocada em um agitador mecânico 


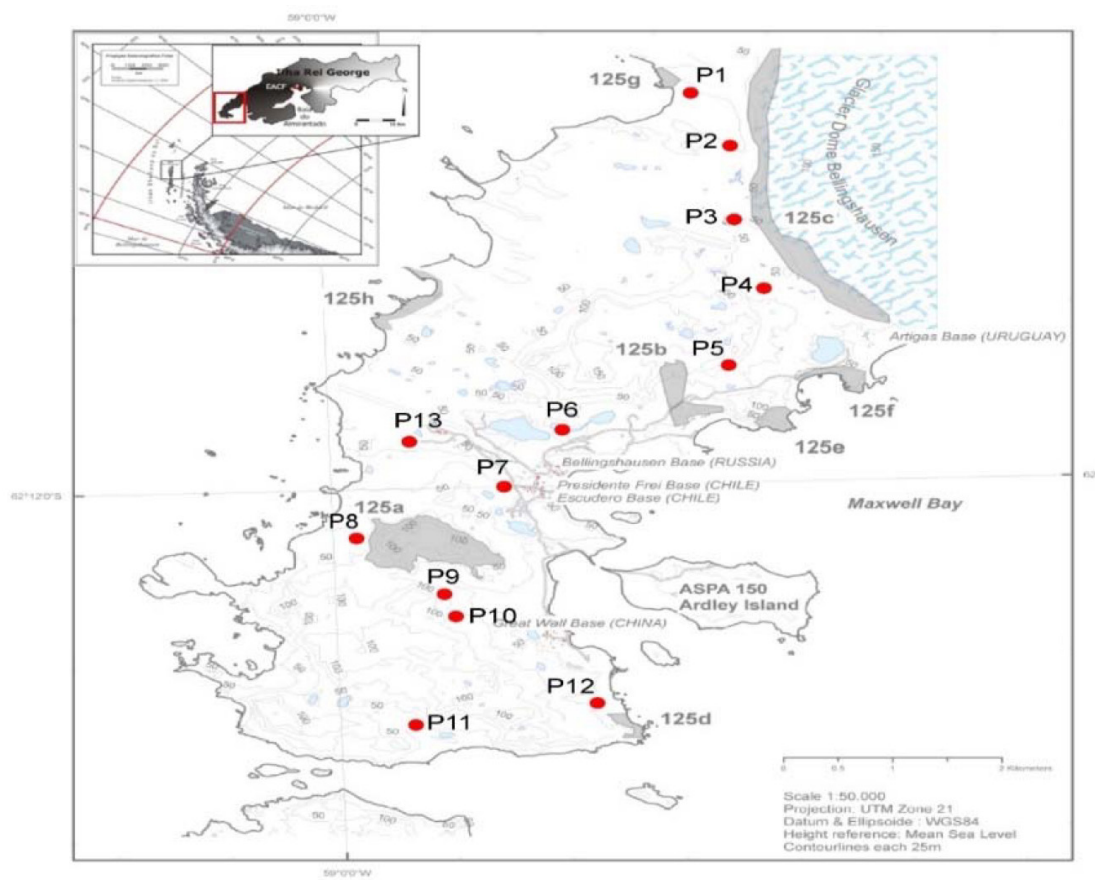

Figura 1. Pontos de coleta de amostras de solo na Península Fildes - Antártica

onde permaneceu por 16 horas a $20^{\circ} \mathrm{C}$; após esse período a solução foi filtrada e transferida para um frasco com capacidade para $50 \mathrm{~mL}$ e acondicionada em geladeira para posterior análise.

O branco de preparo foi submetido ao mesmo procedimento que as amostras. Todas as amostras foram preparadas em triplicata. As curvas analíticas foram construídas a partir de uma solução padrão multielementar, cujo meio foi de acordo com o preparo da amostra, ou seja, $\mathrm{HCl} 2 \%$ v/v. O cálculo da linearidade da curva foi feito por regressão linear.

O procedimento de preparo das amostras para determinação do Fe por F AAS, utilizado nos cálculos de fator de enriquecimento, foi o mesmo utilizado para a determinação dos outros elementos por ICP-MS.

\section{Análises por ICP-MS}

Após o preparo da amostra, os isótopos foram determinados por ICP-MS. Na Tabela 1 estão descritos os parâmetros e condições instrumentais utilizadas na análise.

\section{RESULTADOS E DISCUSSÃO}

\section{Caracterização do solo}

A concentração dos elementos traço em sedimento e solo podem estar relacionados a parâmetros físico-químicos, uma vez que a disponibilidade desses elementos para o meio ambiente depende de fatores tais como o pH que afetam a distribuição, mobilidade e disponibilidade dos elementos. Na Tabela 2 estão dispostos os valores de $\mathrm{pH}$ nas amostras de solo coletada na Ilha Rei George, Península Fildes, Antártica.

Observando os valores de pH (Tabela 2) é possível observar que as amostras de solo apresentam acidez moderada, o que pode
Tabela 1. Condições e parâmetros instrumentais do ICP-MS

Condições e Parâmetros Operacionais

Câmara de nebulização

Ciclônica de vidro com anteparo

Nebulizador

Cones Concêntrico Meinhard, Tipo C

Tocha Níquel 1,1 mm i.d.

Potência da radiofrequência

Introdução da amostra

Fluxo de gás auxiliary

Fluxo de gás de plasma

Fluxo de gás nebulizador

Sweeps

Tocha de quartzo EasyGlide ${ }^{\mathrm{TM}}$

Replicatas

Isótopos mensurados

$1150 \mathrm{~W}$

Bomba peristaltica

$1,20 \mathrm{~L} \mathrm{~min}^{-1}$

$16,00 \mathrm{~L} \mathrm{~min}^{-1}$

$1,1 \mathrm{~L} \mathrm{~min}^{-1}$

45

3

${ }^{208} \mathrm{~Pb},{ }^{114} \mathrm{Cd},{ }^{59} \mathrm{Co},{ }^{118} \mathrm{Sn},{ }^{202} \mathrm{Hg},{ }^{95} \mathrm{Mo}$, ${ }^{75} \mathrm{As}$

Padrão interno

${ }^{103} \mathrm{Rh}$

levar a conclusão de que apenas uma pequena parte dos elementos podem estar retido e/ou adsorvido no solo, uma vez que o $\mathrm{pH}$ ácido possibilita maior mobilidade, disponibilidade e solubilidade dos elementos para o meio. ${ }^{21,22}$

\section{Preparo das amostras de solo}

A prática B, proposta na norma ASTM D3974-09, consistiu no seguinte procedimento: a $1 \mathrm{~g}$ da amostra seca foram adicionados 5,0 $\mathrm{mL}$ de $\mathrm{HCl}$ purificado e concentrado e $95,0 \mathrm{~mL}$ de água purificada; essa mistura foi colocada em um agitador mecânico onde permaneceu

Tabela 2. Valores de pH nas amostras coletadas na Ilha Rei George, Península Fildes, Antártica

\begin{tabular}{lcccccccccc}
\hline Parâmetro/Amostra & P1 & P2 & P3 & P4 & P6 & P8 & P9 & P10 & P11 & P13 \\
\hline $\mathrm{pH}$ & 5,99 & 6,00 & 6,20 & 6,33 & 5,77 & 5,74 & 5,89 & 5,55 & 5,93 & 5,24 \\
\hline
\end{tabular}


por 16 horas; após esse período a solução foi filtrada e transferida para um frasco com capacidade para esse volume e acondicionada em local apropriado.

As soluções obtidas foram analisadas e pôde-se observar pelos resultados que as replicatas apresentaram repetitividade e boas porcentagens de recuperação.

Após a escolha da utilização da ASTM D3974-09 prática B como procedimento de preparo das amostras de solo, optou-se por fazer um novo teste buscando a redução da massa da amostra e do volume dos reagentes utilizados. Para isso reduziu-se pela metade a massa da amostra e o volume dos reagentes, ou seja, utilizou-se $0,5 \mathrm{~g}$ da amostra de solo seca, 2,5 $\mathrm{mL}$ de $\mathrm{HCl}$ purificado e concentrado e 47,5 $\mathrm{mL}$ de água purificada. Observando os resultados obtidos não foi verificada diferença significativa entre os resultados obtidos utilizando a massa descrita no protocolo e a massa reduzida. Desta forma, com o intuito de reduzir o consumo de amostras e reagentes, optou-se por trabalhar com a prática $\mathrm{B}$ em quantidades reduzidas.

\section{Estudo do uso do padrão interno}

Para verificar a necessidade do uso de padrão interno (PI) na determinação de alguns elementos, verificou-se a exatidão do método realizando um teste de recuperação de analito. Por meio dos valores das porcentagens de recuperações obtidas pode-se identificar quais elementos em estudo ( $\mathrm{Pb}, \mathrm{Cd}, \mathrm{Sn}, \mathrm{Co}, \mathrm{Hg}$, Mo e $\mathrm{As}$ ) necessitavam do uso de padrão interno para alguma possível correção.

Os testes de recuperação de analito foram realizados analisando-se os extratos das amostras, obtidas pelo procedimento de preparo $\mathrm{B}$, sem adição e com adição de 5 e $10 \mu \mathrm{g} \mathrm{\textrm {L } ^ { - 1 }}$ dos elementos $\mathrm{Pb}, \mathrm{Cd}$, $\mathrm{Sn}, \mathrm{Co}, \mathrm{Hg}$, Mo e As.

Foram testados os elementos Rh, In e Lu para padronização interna e verificou-se que apenas o $\mathrm{Rh}$ apresentou melhores resultados em relação às determinações. Os resultados obtidos para as determinações sem PI e com Rh como PI podem ser observados na Tabela 3.

De acordo com a Associação Oficial de Química Analítica (AOAC) o valor que pode ser considerado como boa recuperação depende de fatores como a matriz em estudo, o preparo da amostra e a concentração na qual o elemento se encontra. Considerando que os valores dos elementos estudados são de pequena ordem de grandeza, o intervalo aceito como adequado é de 75 a $120 \% .^{23}$
Assim, observando a Tabela 3, pode-se verificar que foram obtidas boas porcentagens de recuperação para o ${ }^{208} \mathrm{~Pb},{ }^{118} \mathrm{Sn}$ e ${ }^{95} \mathrm{Mo}$ sem o uso do padrão interno, indicando ausência de interferências e adequação do procedimento de análise.

Para os dois isótopos de $\mathrm{Cd}$ e $\mathrm{Hg}$, bem como para ${ }^{59} \mathrm{Co}$ e ${ }^{75} \mathrm{As}$ houve indicativo de interferências nas análises dos extratos. Essas interferências podem ser de ordem não espectral e espectral. As interferências não espectrais podem ser identificadas pela perda de sensibilidade da curva analítica e também podem estar relacionadas a problemas de transporte da amostra até o plasma. Essas interferências podem ser minimizadas por meio de equiparação de matriz, diluição da amostra, uso de padrão interno e adição de analito. Já as interferências espectrais ou isobáricas são causadas por íons que apresentam a mesma razão massa/carga do elemento interferindo na intensidade do sinal analítico. Esse tipo de interferência pode ser contornada pela escolha de um isótopo alternativo. ${ }^{24}$

Para os dois isótopos de $\mathrm{Hg}$ obteve-se bons resultados de recuperação com o uso de padrão interno. Como não foi observada diferença significativa entre os dois isótopos, optou-se pelo isótopo ${ }^{202} \mathrm{Hg}$, uma vez que é o mais abundante, com 29,8\%. Assim, adotou-se o uso do PI Rh para as determinações e a utilização do isótopo ${ }^{202} \mathrm{Hg}$.

Para o As também foram observados melhores valores de recuperação utilizando padrão interno Rh, que provavelmente minimizou as interferências não espectrais que podem ocorrer para este isótopo.

$\mathrm{Na}$ determinação do $\mathrm{Cd}$ pôde-se observar melhores porcentagens de recuperação com o uso de PI. Comparando os dois isótopos, observou-se que $\mathrm{o}^{114} \mathrm{Cd}$ apresentou melhores valores de recuperação quando comparado ao ${ }^{112} \mathrm{Cd}$, ficando estabelecido a utilização do ${ }^{114} \mathrm{Cd}$ com padrão interno Rh para as determinações.

Para Co verificou-se que as recuperações obtidas encontraram-se fora da faixa considerada adequada $(75-120 \%) .{ }^{23}$ Ao utilizar o PI Rh, melhores valores foram obtidos, mas ainda estão abaixo da faixa de aceitação. Para este elemento foi verificado que a equiparação de matriz, a adição de analito e o uso de padrão interno não contornaram as possíveis interferências. Sugere-se, então, que os valores de recuperação não satisfatórios estejam relacionados às interferências espectrais, uma vez que este elemento apresenta baixa relação massa/ carga quando comparado aos demais elementos. Porém, devido ao isótopo ${ }^{59} \mathrm{Co}$ apresentar abundância de $100 \%$, não foi possível estudar outra razão isotópica em busca de melhores resultados.

Tabela 3. Valores de recuperação (\%) obtidos nas amostras de extratos de solos da Antártica analisadas por ICP-MS

\begin{tabular}{|c|c|c|c|c|c|c|c|c|c|c|}
\hline & \multirow{2}{*}{ Amostra } & \multicolumn{9}{|c|}{ RECUPERAÇÃO (\%) } \\
\hline & & ${ }^{208} \mathrm{~Pb}$ & ${ }^{114} \mathrm{Cd}$ & ${ }^{112} \mathrm{Cd}$ & ${ }^{118} \mathrm{Sn}$ & ${ }^{59} \mathrm{Co}$ & ${ }^{202} \mathrm{Hg}$ & ${ }^{200} \mathrm{Hg}$ & ${ }^{95} \mathrm{Mo}$ & ${ }^{75}$ As \\
\hline \multirow{6}{*}{ Sem PI } & $\mathrm{P} 11^{1}$ & 81,6 & 76,4 & 58,7 & 86,4 & 138,4 & 63,0 & 64,5 & 93,2 & 126,7 \\
\hline & $\mathrm{P} 11^{2}$ & 93,5 & 85,3 & 14,4 & 93,5 & - & 79,7 & 80,0 & 100,9 & - \\
\hline & $\mathrm{P}^{1}$ & 91,8 & 72,0 & 65,7 & 82,4 & 253,5 & 74,1 & 74,3 & 99,3 & 145,2 \\
\hline & $\mathrm{P}^{2}$ & 91,2 & 73,2 & 68,1 & 84,6 & - & 77,7 & 78,1 & 99,2 & - \\
\hline & $\mathrm{P} 9^{1}$ & 99,1 & 77,1 & 71,7 & 89,4 & 210,8 & 83,2 & 83,8 & 100,4 & 231,6 \\
\hline & $\mathrm{P}^{2}$ & 100,0 & 81,6 & 76,7 & 94,8 & - & 88,0 & 88,6 & 107,3 & - \\
\hline \multirow{6}{*}{ Com Rh } & $\mathrm{P} 11^{1}$ & 83,9 & 85,9 & 74,3 & 96,4 & 70,9 & 90,6 & 92,9 & 104,3 & 93,8 \\
\hline & $\mathrm{P} 11^{2}$ & 139,6 & 116,6 & 31,5 & 129,0 & - & 121,9 & 123,1 & 137,5 & - \\
\hline & $\mathrm{P}^{1}$ & 134,2 & 98,3 & 89,8 & 113,5 & 75,1 & 103,7 & 105,0 & 135,2 & 101,5 \\
\hline & $\mathrm{P} 6^{2}$ & 132,1 & 101,1 & 94,1 & 117,7 & - & 108,4 & 109,8 & 136,9 & - \\
\hline & $\mathrm{P} 91$ & 89,9 & 81,0 & 75,2 & 90,7 & 70,7 & 90,4 & 90,8 & 106,6 & 96,7 \\
\hline & $\mathrm{P} 9^{2}$ & 79,8 & 75,3 & 70,6 & 84,6 & - & 82,8 & 83,4 & 99,7 & - \\
\hline
\end{tabular}

${ }^{1}$ adição de $5 \mu \mathrm{g} \mathrm{L}{ }^{-1}{ }^{2}$ adição de $10 \mu \mathrm{g} \mathrm{L}{ }^{-1}$. 


\section{Figuras analíticas de mérito}

Para a verificação do procedimento analítico foram avaliados alguns parâmetros e condições de análise que são utilizados para atestar a qualidade do seu método. Neste trabalho foram abordados três parâmetros (linearidade do método, LD e LQ), além do ensaio de adição de analito e recuperação.

Para a avaliação da linearidade do método construiu-se curva analítica para cada elemento estudado e por meio de regressão linear obteve-se o coeficiente de determinação $\left(\mathrm{R}^{2}\right)$. Os valores de coeficiente de determinação variaram de 0,9993 a 0,9998. Visto que a Anvisa recomenda que a curva analítica tenha um $\mathrm{R}^{2}$ maior ou igual a 0,999 , pode-se inferir que o método proposto apresentou uma boa linearidade para todos os elementos. ${ }^{25}$

Os valores de limite de detecção (LD) e limite de quantificação (LQ) foram obtidos a partir da análise do branco de preparo. Para o cálculo desses dois parâmetros foram utilizadas as definições propostas pela IUPAC $(\mathrm{LD}=3 \mathrm{~s} / \mathrm{m}$ e LQ $=10 \mathrm{~s} / \mathrm{m}$, em que $\mathrm{s}$ é o desvio padrão estimado de 10 leituras do branco de preparo e m é a inclinação da curva analítica). Os resultados obtidos para esses dois parâmetros estão dispostos na Tabela 4 e são adequados para a análise dos extratos de solo.

Como já apresentado na Tabela 3, os valores de recuperação obtidos foram satisfatórios (75 a 120\%), ${ }^{24}$ indicando uma boa exatidão do método para a determinação de $\mathrm{Pb}, \mathrm{Cd}, \mathrm{Sn}, \mathrm{Hg}$, Mo e As. Apenas para o Co, utilizando Rh como PI, os valores de recuperação apresentaram-se um pouco abaixo da faixa adequada.

Tabela 4. Limites de detecção (LD) e de quantificação (LQ) do método de determinação e nas amostras

\begin{tabular}{ccccc}
\hline Isótopo & $\begin{array}{c}\text { LD do } \\
\text { método } \\
\left(\mu \mathrm{g} \mathrm{L}^{-1}\right)\end{array}$ & $\begin{array}{c}\text { LD na } \\
\text { amostra } \\
\left(\mu \mathrm{g} \mathrm{kg}^{-1}\right)\end{array}$ & $\begin{array}{c}\text { LQ do } \\
\text { método } \\
\left(\mu \mathrm{g} \mathrm{L}^{-1}\right)\end{array}$ & $\begin{array}{c}\text { LQ na } \\
\text { amostra } \\
\left(\mu \mathrm{kg}^{-1}\right)\end{array}$ \\
\hline${ }^{208} \mathrm{~Pb}$ & 0,011 & 1,1 & 0,035 & 3,5 \\
${ }^{114} \mathrm{Cd}$ & 0,0021 & 0,21 & 0,0069 & 0,69 \\
${ }^{118} \mathrm{Sn}$ & 0,24 & 24 & 0,79 & 79 \\
${ }^{59} \mathrm{Co}$ & 0,0029 & 0,29 & 0,0097 & 0,97 \\
${ }^{202} \mathrm{Hg}$ & 0,024 & 2,4 & 0,081 & 8,1 \\
${ }^{95} \mathrm{Mo}$ & 0,053 & 5,3 & 0,17 & 17 \\
${ }^{75} \mathrm{As}$ & 0,72 & 72 & 2,4 & 238 \\
\hline
\end{tabular}

Determinação e avaliação das concentrações de $\mathrm{Pb}, \mathrm{Cd}, \mathrm{Sn}, \mathrm{Co}$, Hg, Mo e As nas amostras de solo da Antártica

Os extratos das amostras de solo, obtidos utilizando o procedimento de extração (ASTM D3974-09) em triplicata, foram analisados por ICP-MS com as condições otimizadas anteriormente. A média dos resultados de concentração encontrados nas replicatas para cada elemento nas amostras coletadas nos diferentes pontos da Península Fildes - Antártica estão dispostos na Tabela 5. Observou-se que não há uma distribuição homogênea dos elementos por toda a extensão da Península Fildes.

Utilizando os valores apresentados na Tabela 5 e o programa Surfer versão 8 Free Demo (Golden Software) foi realizada uma estimativa da distribuição de cada elemento nos solo coletados em toda Península Fildes.

Para o $\mathrm{Pb}$ pode-se observar que maiores valores são encontrados próximos a locais que recebem afluentes de degelo recente - amostras P3 e P4 (Figura 2). Era esperado que as amostras coletadas próximo a essa região de degelo recente apresentariam menores concentrações, uma vez que a região esteve coberta pelo gelo até poucos anos atrás, e isto foi observado para amostras P1 e P2. Porém, um evento provável de ocorrer é o processo de deposição atmosférica ocorrido na superfície da geleira, que torna possível o acúmulo ao longo de anos, e devido o processo de retração dessa geleira pode haver uma redisponibilização os materiais acumulados. Vale ressaltar que próximo a esses pontos de coleta existe um rio proveniente do degelo tornando possível que pontos considerados intocados apresentem concentrações significativas de metal, ${ }^{26,27}$ o que pode justificar os maiores teores de $\mathrm{Pb}$ encontrados nas amostras $\mathrm{P} 3$ e $\mathrm{P} 4$.

Para Cd verifica-se um comportamento de distribuição semelhante ao $\mathrm{Pb}$ (Figura 2).

Estudo semelhante em solos da Península Fildes foi proposto por Amaro e colaboradores, ${ }^{28}$ no qual obtiveram concentração de $\mathrm{Pb}$ igual a $6200 \mu \mathrm{g} \mathrm{kg}^{-1}$ e Cd igual a $400 \mu \mathrm{g} \mathrm{kg}^{-1}$, superior aos valores encontrados nesse trabalho (525-2314 $\mu \mathrm{g} \mathrm{kg}^{-1}$ para $\mathrm{Pb}$ e $55-194 \mu \mathrm{g} \mathrm{kg}^{-1}$ para Cd). Essa diferença de concentração observada pode ser devido aos diferentes procedimentos de digestão aplicados em cada trabalho, que pode influenciar na disponibilidade do elemento para quantificação, bem como a diferença dos pontos de coleta.

No estudo da distribuição de Sn, observa-se pela Figura 3 que há duas regiões onde se concentram as amostras com maiores quantidades desse elemento, os pontos 3, 4 e 5 e os pontos 8, 9 e 10. Existe a possibilidade desses dois locais estarem recebendo uma carga de Sn

Tabela 5. Concentração dos elementos, e suas respectivas incertezas, encontradas nas amostras de solo coletadas nos diferentes pontos da Península Fildes Antártica

\begin{tabular}{|c|c|c|c|c|c|c|c|}
\hline \multirow{2}{*}{ Amostra } & \multicolumn{7}{|c|}{ Concentração $\left(\mu \mathrm{g} \mathrm{kg}^{-1}\right)$} \\
\hline & $\mathrm{Pb}$ & $\mathrm{Cd}$ & Sn & $\mathrm{Co}$ & $\mathrm{Hg}$ & Mo & As \\
\hline $\mathrm{P} 1$ & $530 \pm 1$ & $184 \pm 7$ & $110 \pm 3$ & $2887 \pm 28$ & $<2,4$ & $<5,3$ & $4517 \pm 177$ \\
\hline $\mathrm{P} 2$ & $610 \pm 26$ & $194 \pm 9$ & $324 \pm 18$ & $2970 \pm 11$ & $13 \pm 2$ & $<5,3$ & $7866 \pm 227$ \\
\hline P3 & $1488 \pm 7$ & $55 \pm 1$ & $1374 \pm 61$ & $8037 \pm 751$ & $24,7 \pm 0,6$ & $38,7 \pm 0,5$ & $11539 \pm 60$ \\
\hline $\mathrm{P} 4$ & $2314 \pm 5$ & $106 \pm 1$ & $1087 \pm 8$ & $5500 \pm 173$ & $28,4 \pm 0,5$ & $11,4 \pm 0,5$ & $5851 \pm 82$ \\
\hline P6 & $777 \pm 19$ & $107 \pm 3$ & $447 \pm 14$ & $6251 \pm 32$ & $29,7 \pm 0,2$ & $17 \pm 5$ & $4553 \pm 165$ \\
\hline P8 & $733 \pm 20$ & $79 \pm 5$ & $928 \pm 52$ & $11094 \pm 103$ & $42 \pm 2$ & $11,2 \pm 0,4$ & $8123 \pm 499$ \\
\hline P9 & $525 \pm 23$ & $56 \pm 6$ & $929 \pm 93$ & $4598 \pm 50$ & $24,7 \pm 0,3$ & $9 \pm 2$ & $6123 \pm 47$ \\
\hline P10 & $549 \pm 37$ & $64 \pm 1$ & $774 \pm 85$ & $1782 \pm 17$ & $26,2 \pm 0,9$ & $15 \pm 1$ & $5132 \pm 45$ \\
\hline P11 & $724 \pm 23$ & $64 \pm 2$ & $437 \pm 40$ & $2095 \pm 2$ & $39 \pm 10$ & $4,1 \pm 0,6$ & $4852 \pm 381$ \\
\hline $\mathrm{P} 13$ & $840 \pm 3$ & $67 \pm 3$ & $530 \pm 3$ & $5264 \pm 22$ & $55 \pm 1$ & $<5,3$ & $6806 \pm 21$ \\
\hline Intervalo de concentração & $525-2314$ & $55-194$ & $110-1374$ & $1782-11094$ & $<2,4-55$ & $<5,3-38,7$ & $4517-11539$ \\
\hline
\end{tabular}



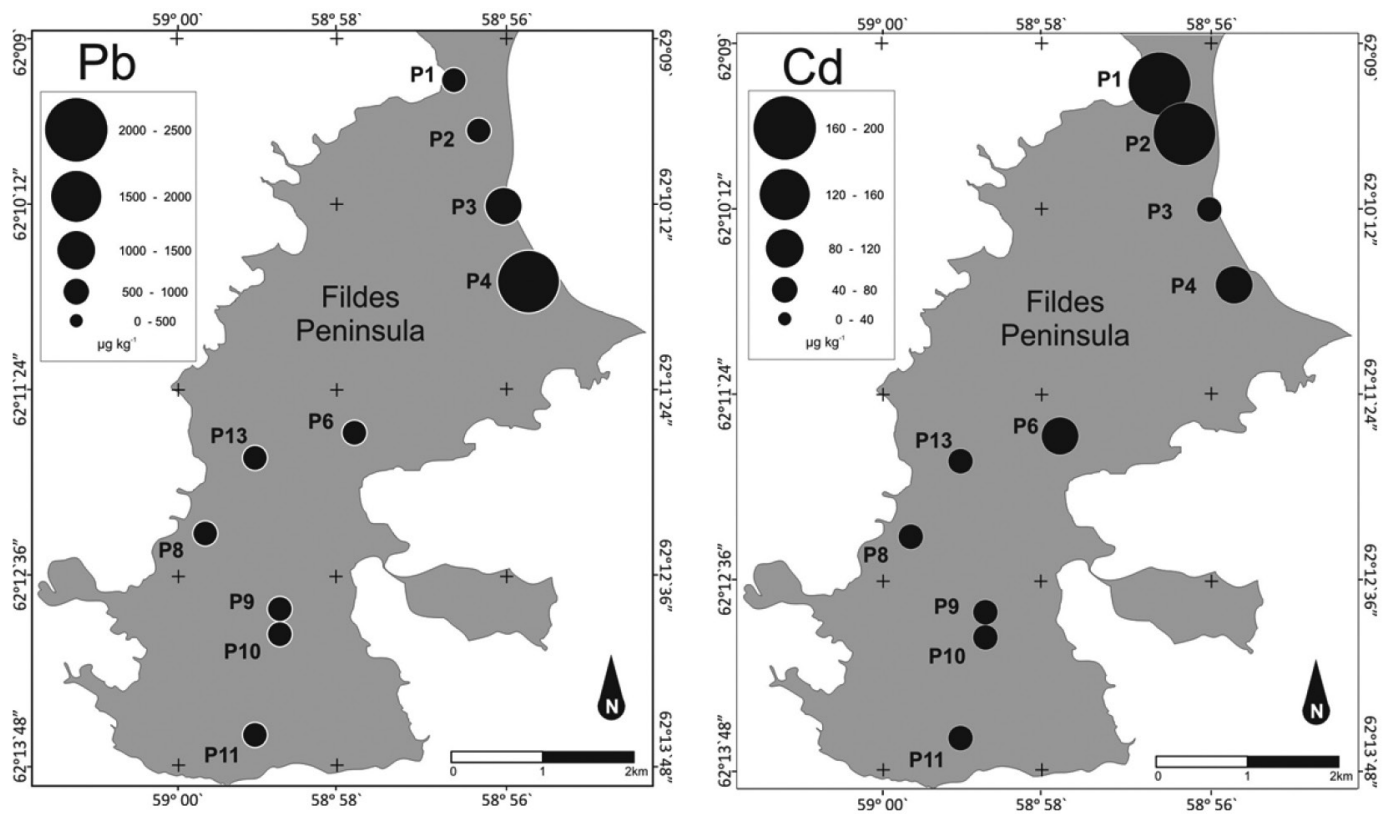

Figura 2. Distribuição da concentração de Pb e de Cd na Península Fildes - Antártica

devido à existência de estações de pesquisa próximas, como a estação Artigas do Uruguai, localizada entre os pontos 4 e 5, e a estação Great Wall pertencente à China, localizada entre os pontos 9 e 10. Além disso, o ponto 8 fica próximo à única pista de pouso da ilha.

Para a distribuição de $\mathrm{Hg}$ verificou-se uma maior concentração na região próxima aos pontos 6,7, 8, 11 e 13 (Figura 3). As concentrações nas amostras variaram de $<2,4$ a $55 \mu \mathrm{g} \mathrm{kg}^{-1}$, encontrando-se dentro da faixa já reportada na literatura para a mesma região. No trabalho de Santos e colaboradore ${ }^{29}$ foi encontrada uma concentração de 24,6 $\mu \mathrm{g} \mathrm{kg}^{-1}$ em sedimento, enquanto que Lu e colaboradores, ${ }^{12}$ em seus estudos, encontraram concentrações que variaram entre $10 \mathrm{a}$ $60 \mu \mathrm{g} \mathrm{kg}^{-1}$. É interessante salientar a existência de uma pista de pouso com intensa movimentação de carga e descarga de materiais, bem como de transporte de pessoas, próxima aos pontos 8 e 13 .

As concentrações encontradas para o Mo em relação aos demais elementos são as menores, apresentando a maior concentração o ponto 3 (Figura 4). Assim como citado anteriormente, pode-se atribuir essa maior concentração na região em que é cortada por um rio de degelo. Santos e colaboradores,${ }^{10}$ em estudos em amostras de sedimento coletados em outra ilha da Antártica, encontraram concentração desse elemento igual a $1500 \mu \mathrm{g} \mathrm{kg}^{-1}$, superior à encontrada nesse estudo $\left(<5,3-38,7 \mu \mathrm{g} \mathrm{kg}^{-1}\right)$. Tal diferença pode ser atribuída ao fato dos estudos terem sido realizados em diferentes locais do continente.

Na distribuição de As observa-se que grande parte da Península apresentou valores semelhantes de concentração, indicando que há para esse elemento uma distribuição mais uniforme quando comparado aos outros elementos (Figura 4). Amaro et al. ${ }^{28}$ encontraram concentração de As igual a $3800 \mu \mathrm{g} \mathrm{kg}^{-1}$ em amostras de solo da Península Fildes, enquanto que Ribeiro et al. ${ }^{29}$ estudaram a concentração de As na Baía Almirantado-Antártica e relataram uma faixa de concentração de $2000 \mathrm{a} 12000 \mu \mathrm{g} \mathrm{kg}^{-1}$, semelhante à faixa encontrada neste estudo (4517 a $11539 \mu \mathrm{g} \mathrm{kg}^{-1}$ ).
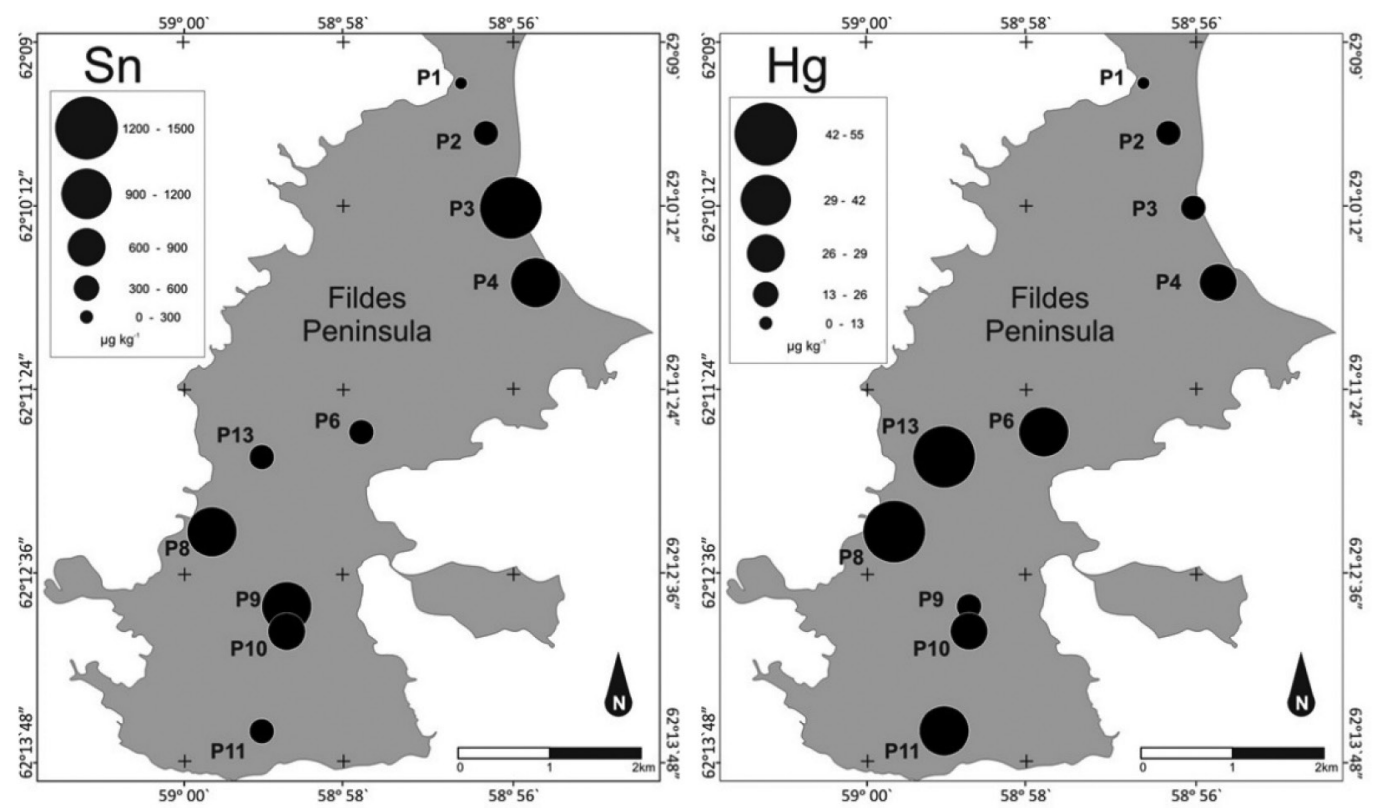

Figura 3. Distribuição da concentração de Sn e de Hg na Península Fildes - Antártica 

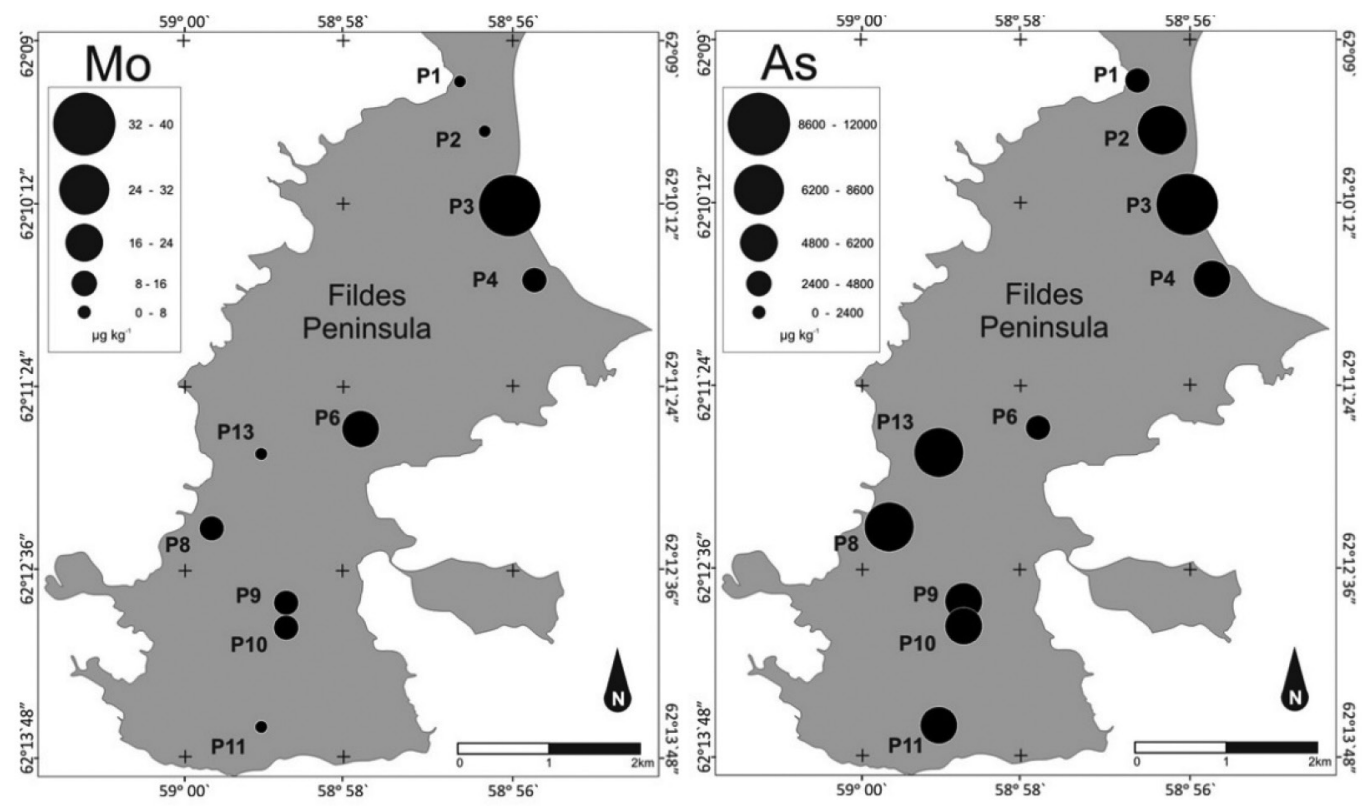

Figura 4. Distribuição da concentração de Mo e de As na Península Fildes - Antártica

Pela análise da Figura 5, verificou-se que o Co apresentou maiores concentrações na amostra coletada no ponto 8. Isso pode ter ocorrido uma vez que existem estações de pesquisas próximas a esse local, bem como uma pista de pouso.

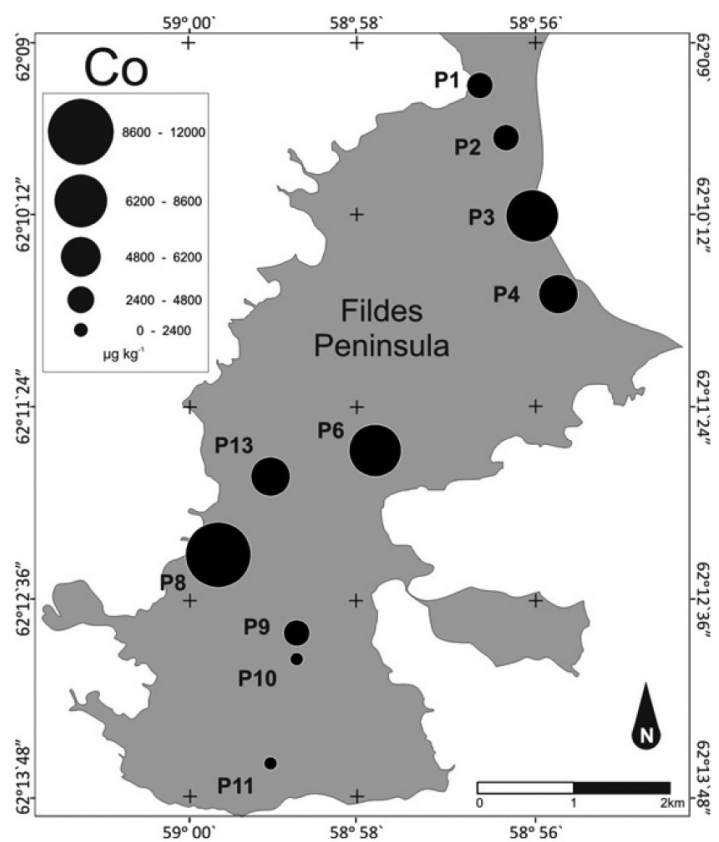

Figura 5. Distribuição da concentração de Co na Península Fildes - Antártica

Normalmente maiores valores de concentração seriam esperados para a região central da Península, uma vez que nessa região está localizada uma pista de pouso com intensa movimentação de pessoas e cargas, principalmente no verão, assim como uma base militar e duas estações de pesquisa. Apesar disso, os elementos determinados apresentaram, de um modo geral, valores distribuídos por toda a superfície da península, o que pode estar associado a diversos fatores, tais como presença natural, correntes de ar e marítimas, processos de degelo, bem como as atividades antrópicas locais.

Diversos trabalhos já reportaram o transporte em nível regional e global e deposição local de diversos elementos químicos. ${ }^{13}$ Nesse processo de deposição, as maiores áreas de recepção são as superfícies das geleiras, por apresentarem uma superfície aérea muito maior do que as áreas livres de gelo, como é o caso da Península Fildes. Portanto, pode existir uma tendência maior de acúmulo na matriz gelo do que em solos.

Um processo que vem sendo observado no último século na região da Península Antártica é a elevação local das temperaturas e retração das geleiras, que pode redisponibilizar para o ambiente materiais que foram incorporados à matriz do gelo ao longo dos anos..$^{30,31}$ Esse processo é normalmente mais intenso na região frontal da geleira, onde se formam rios de degelo que concentram a passagem de toda água produzida no processo. Esse processo é compatível com comportamento das distribuições dos metais $\mathrm{Pb}, \mathrm{Cd}$, Sn e As encontradas neste trabalho. Além disso, a Península Antártica naturalmente é uma região de ocorrência de ciclones, o que faz com que ventos de grande intensidade ocorram frequentemente. Este fenômeno pode ter contribuído para a distribuição heterogênea dos elementos $\mathrm{Co}, \mathrm{Hg}$ e Mo ao longo da Península Fildes.

\section{Fator de enriquecimento}

Com o intuito de auxiliar na avaliação dos possíveis impactos no solo da Península Fildes, foi utilizado o fator de enriquecimento (FE) como uma ferramenta para estimar se os valores de concentração encontrados dos elementos podem ser de origem antropogênica. $\mathrm{O}$ FE pode ser definido como sendo a razão entre a concentração do elemento em estudo e a concentração do elemento normalizador dividido pela razão da concentração referência do elemento em estudo e da concentração referência do elemento normalizador. ${ }^{10,32}$ Elementos como Fe, $\mathrm{Al} \mathrm{e} \mathrm{Li} \mathrm{têm} \mathrm{sido} \mathrm{comumente} \mathrm{utilizados} \mathrm{como} \mathrm{normalizadores}$ geoquímicos. No presente estudo utilizou-se o Fe como elemento normalizador e os valores de referência para os elementos analisados foram extraídos da literatura. ${ }^{33}$

As faixas dos valores de $\mathrm{FE}$ foram calculadas para $\mathrm{Pb}, \mathrm{Cd}, \mathrm{Co}$ e $\mathrm{As},{ }^{33}$ uma vez que na literatura não foram encontrados valores de referência de concentração para os outros elementos em estudo utilizando o mesmo procedimento de preparo de amostra. Os valores calculados foram: $3,2 \times 10^{-5}$ a 0,01 para $\mathrm{Pb} ; 0$ para $\mathrm{Cd}$; 42,6 a 85,9 para Co e 30,6 a 107,9 para As. Esses valores obtidos foram comparados com a classificação que divide o FE em categorias que 
inferem os níveis de poluição antrópica. ${ }^{32} \mathrm{Para} \mathrm{Pb}$ e $\mathrm{Cd}$ foram obtidos valores de $\mathrm{FE}<2$, sugerindo que não há contribuição antrópica ou a contribuição é mínima em relação a esses elementos; porém, para Co e As foram obtidos valores de FE mais elevados, FE $>40$, indicando alta contribuição antropogênica.

\section{CONCLUSÕES}

Verificou-se que o procedimento de extração dos elementos das amostras de solo (ASTM 3974-09 prática B modificada) apresentou boa repetitividade. Em relação ao método de determinação dos elementos foi possível verificar, por meio dos testes de adição de analito e recuperação, uma exatidão satisfatória para $\mathrm{Pb}$, Sn e Mo sem a utilização de padrão interno, enquanto que para $\mathrm{Cd}$, $\mathrm{As}$, $\mathrm{Co}$ e $\mathrm{Hg}$ fez-se necessário o uso de $\mathrm{Rh}$ como padrão interno para corrigir possíveis interferências não espectrais. Além disso, o método apresentou boa linearidade $\left(R^{2}>0,999\right)$ e valores de limite de detecção e quantificação apropriados às determinações dos elementos em estudo nos extratos. Deste modo, pode-se inferir que a metodologia proposta de preparo e análise das amostras de solo da Antártica mostrou-se adequada.

Observou-se através dos resultados obtidos que há uma tendência, principalmente para $\mathrm{Pb}, \mathrm{Cd}$, $\mathrm{Sn}$ e As, de apresentarem concentrações mais expressivas nas amostras de solo coletadas em local de degelo recente.

Embora fossem esperados valores de concentração mais elevados nas amostras coletadas na região central da Península, devido à presença de uma pista de pouso com intensa movimentação de pessoas e cargas, uma base militar e duas estações de pesquisa, isto não foi observado neste estudo. De um modo geral os valores de concentrações dos elementos se apresentaram distribuídos por toda a superfície da península, o que pode estar associado a diversos fatores, tais como presença natural, correntes de ar e marítimas, processos de degelo, bem como as atividades antrópicas locais.

Utilizando o fator de enriquecimento, verificou-se que para o As e Co existe um alto indicativo de contribuição antropogênica nas amostras estudadas, enquanto que para $\mathrm{Pb}$ e $\mathrm{Cd}$ não há indicativo. Deste modo, é importante salientar que os níveis dos elementos devem ser constantemente monitorados, possibilitando realizar algumas inferências quanto à origem desses elementos e auxiliar na proteção ambiental.

\section{REFERÊNCIAS}

1. Bargagli, R.; Sci. Total Environ. 2008, 400, 212.

2. Gasparon, M.; Matschullat, J.; Appl. Geochem. 2006, 21, 1593.

3. Celis, J.; Jara, S.; Acuña, G. D.; Barra, R.; Espejo, W.; Arch. Med. Vet. 2012, 44, 311.

4. Leal, M. A.; Joppert, M.; Licínio, M. V.; Evangelista, H.; Maldonado, J.; Dalia, K. C.; Lima. C.; Leite, C. V. B.; Correa, S. M.; Medeiros, G.; Cunha, K. D.; Water Air Soil Pollut. 2008, 188, 67.

5. Martins, C. C.; Aguiar, S. N.; Bícego, M. C.; Montone, R. C.; Mar. Pollut. Bull. 2012, 64, 2867.

6. Vodopivez, C.; Smichowski, P.; Marcovecchio, J.; Environ. Contam. Antarct. 2001, 6, 156.
7. Cipro, C. V. Z.; Yigui, G. T.; Bustamante, P.; Taniguchi, S.; Sericano, J. L.; Montone, R. C.; Chemosphere 2011, 85, 393.

8. Jerez, S.; Motas, M.; Benzal, J.; Diaz, J.; Barbosa, A.; Mar. Pollut. Bull. 2013, 69, 67.

9. Verllinger, C.; Parant, M.; Rousselle, P.; Immel, F.; Wagner, P.; Polatera, P. U.; Environ. Pollut. 2012, 160, 66.

10. Santos, I. R.; Silva Filho, E. V.; Schaefer, C. E. G. R.; Albuquerque Filho, M. R, L. S.; Mar. Pollut. Bull. 2005, 50, 185.

11. Tao, Y.; Yuan, Z.; Xiaona, H.; Wei, M.; Ecotoxicol. Environ. Saf. 2012, $81,55$.

12. Lu, Z.; Cai, M.; Wang, J.; Yang, H.; He, J.; Environ. Monit. Assess. 2012, $184,7013$.

13. Licínio, M. V.; Patchineelan, S. R.; Evangelista, H. S.; Araripe, D. R.; Indian J. Mar. Sci. 2008, 37, 391.

14. Figueroa, D. A.; Jimenez, B. D.; Sierra, C. J. R.; Environ. Pollut. 2006, $141,336$.

15. Sastre, J.; Sahuquillo, A.; Vidal, M.; Rauret, G.; Anal. Chim. Acta 2002, $462,59$.

16. Nascentes, C. C.; Arruda, M. A. Z.; Nogueira, A. R. A.; Nóbrega, J. A.; Talanta 2004, 64, 912.

17. Pereira, L. A.; Amorim, I. G.; Silva, J. B. B.; Talanta 2004, 64, 395.

18. Mouli, P. C; Mohan, S. V.; Balaram, V.; Kumar, M. P.; Reddy, S, J.; Atmos. Environ. 2006, 40, 136.

19. Amaral, A. A.; Pires, S. C.; Ferrari, J. L.; Agroambiente 2014, 8, 194.

20. ASTM Internacional; Standart practices for extraction of trace elements from sediments, D3974 -09. United States, 2009.

21. Jesus, H. C.; Costa, E. A.; Mendonça, A. S. F.; Zandonade, E.; Quim. Nova 2004, 27, 378.

22. Martins, C. A. da S.; Nogueira, N. O.; Ribeiro, P. H.; Rigo, M. M.; Candido, A. O.; Revista Brasileira de Agrociência 2011, 383.

23. AOAC Official Methods of Analysis; Guidelines for standard method performance requirements, 2012.

24. Nunes, J. A.; Dissertação de mestrado, Universidade de São Paulo, Brasil, 2009.

25. ANVISA; Portaria $n^{\circ}$ 685, de 27 de agosto de 1998. Disponível em: http://www.anvisa.gov.br/legis/portarias/685_98.htm, acessada em setembro de 2015.

26. Gulin, S. B.; Stokozov, N. A.; J. Environ. Radioact. 2005, 84, 1.

27. Sanders, C. J.; Santos, I. R.; Patchineelam, S. R.; Schaefer, C.; SilvaFilho, E. V.; J. Environ. Radioact. 2010, 101, 421.

28. Amaro, E.; Padeiro, A.; Ferro, A. M.; Mota, A. M.; Leppe, M.; Verkulich, S.; Hghes, K. A.; Peter, H. U.; Canário, J.; Mar. Pollut. Bull. 2015, 97, 523 .

29. Santos, I. R.; Silva-Filho, E. V.; Schaefer, C.; Sella, S. M.; Silva, C. A.; Gomes, V.; Passos, M. J. A. C. R.; Ngan, P. V.; Environ. Pollut. 2006, 140,304

30. Mulligan, C. N.; Fukue, M.; Sato, Y.; Sediments contamination and sustainable remediation, $1^{\text {st }}$ ed., CRC Press: London, 2009.

31. Ribeiro, A. P.; Figueira, R. C.; Martins, C. C.; Silva, C. R. A.; França, E. J.; Bícego, M. C.; Mahiques, M. M.; Montone, R. C.; Mar. Pollut. Bull. 2011, 62, 192.

32. Sutherland, R. A.; Environ. Geol. 2000, 39, 611.

33. Gasparon, M.; Matschullat, J.; Appl. Geochem. 2006, 21, 1596. 